\title{
Understanding the factors involved in determining the bioburdens of surgical masks
}

\author{
Zhiqing Liu", Degang Yu\#, Yuwei Ge, Liao Wang, Jingwei Zhang, Huiwu Li, Fengxiang Liu, Zanjing Zhai \\ Shanghai Key Laboratory of Orthopaedic Implants, Department of Orthopaedic Surgery, Shanghai Ninth People's Hospital, Shanghai Jiao Tong \\ University School of Medicine, Shanghai 200011, China \\ Contributions: (I) Conception and design: Z Zhai; (II) Administrative support: F Liu; (III) Provision of study materials or patients: F Liu; \\ (IV) Collection and assembly of data: Z Liu, D Yu, Y Ge, L Wang, J Zhang, H Li; (V) Data analysis and interpretation: Z Zhai, F Liu; \\ (VI) Manuscript writing: All authors; (VII) Final approval of manuscript: All authors. \\ \#These authors contributed equally to this work. \\ Correspondence to: Zanjing Zhai. Shanghai Key Laboratory of Orthopaedic Implants, Department of Orthopaedic Surgery, Shanghai Ninth People's \\ Hospital, Shanghai Jiao Tong University School of Medicine, 639 Zhizaoju Road, Shanghai 200011, China. Email: zanjing_zhai@163.com; \\ Fengxiang Liu. Department of Orthopaedics, Ninth People's Hospital, Shanghai Jiao Tong University School of Medicine, Shanghai, China. Email: \\ liu_fengxiang@126.com.
}

Background: Surgical site infection (SSI) continues to be one of the most common postoperative complications. In our previous study, surgical mask (SM) bioburden was identified to be a potential source of SSI. In the present study, we investigated the factors involved in SM bioburden.

Methods: Bioburdens of the disposable SM (A: medical mask; B: medical surgical mask) and newly laundered cloth SM (C) were tested by immediately making an impression of the external surface of the mask on sterile culture media. SM microstructure was observed using a scanning electron microscope (SEM). Filtering efficiency and airflow resistance were evaluated with TSI Automated Filter Tester 8130 (TSI Incorporated) according to GB/19083-2010. Whether speaking during operation and washing the face pre-operatively affect SM bioburdens was also evaluated. Surgical procedures were performed in a dynamic operation room. Fifty cases of mask use were enrolled in this study.

Results: The bioburden of mask A was the highest. The bioburden of mask B was the lowest. Mask C possessed the lowest filtering efficiency and the highest airflow resistance. SM bioburden was higher in the speaking group. SM bioburden showed no significant difference after washing the face, despite the finding that washing could significantly reduce facial bioburden.

Conclusions: Multiple factors influence SM bioburdens. Mask B showed the lowest bioburden and best protection effects. Mask $\mathrm{C}$ is not recommended to be used, especially considering that surgeons do not wash the cloth masks daily. Unnecessary talking during operation is not recommended, and washing the face before surgery is not strictly necessary.

Keywords: Surgical mask (SM); bioburden; mask type; speaking; washing face

Submitted Jul 18, 2019. Accepted for publication Oct 25, 2019.

doi: $10.21037 /$ atm.2019.11.91

View this article at: http://dx.doi.org/10.21037/atm.2019.11.91

\section{Introduction}

Surgical site infection (SSI) continues to be one of the most common postoperative complications; it is associated with significantly increased morbidity and mortality, prolonged hospital stays, and added costs, all of which cause substantial clinical and economic burdens (1-3). Determining effective strategies to prevent and/or control SSI is therefore vital.

Regarding SSI, airborne contamination and microbial shedding from personnel have been identified as the potential infectious sources (4). Effective ventilation strategies and 
surgical attire have been applied in modern operating rooms to counter these. To some extent, reducing air-loaded contamination and microbial shedding during surgeries may help prevent/control SSIs (5-7). Therefore, surgeons are required to wear surgical attire during operations, including a surgical gown, sterile gloves, headgear, and masks, which together attempt to create a physical barrier between the surgeons and the patients (8). However, only the use of gloves and impervious surgical gowns has been found to reduce SSI. It was demonstrated that the use of masks and head coverings reduced contamination in operating rooms; however, the prevalence of SSI did not decline accordingly (8).

Surgical masks (SMs) were first developed in the early 20th century, after which they became the standard operating apparel and are now used routinely (9). It was observed that SMs significantly reduced the bacterial dispersal rates directly in front of the mouth (10), while other studies showed that the use of SMs failed to reduce the overall operating room bacterial counts (11). Over the past decades, whether SMs can decrease the rate of SSIs has been called into question since present research has been unable to identify the pronounced effect of SMs in reducing SSIs (12-14). However, current Occupational Safety and Health Administration (OSHA) regulations and universal precautions require face masks as part of personal protective equipment for scrubbed personnel (8).

In our previous study, we confirmed that the bioburden of SMs increase with prolonged wearing time, and SMs are a potential source of bacterial shedding, which may increase the risk of SSIs (15). Notably, in prolonged and complicated surgical procedures such as total joint arthroplasty (TJA), we observed that large amounts of blood and dust were produced when the pendulum saw was used to cut off bone, which splashed on the surface of SMs and rebounded to the operative field (15). Thus, strategies that could decrease SM bioburdens may help to decrease bacterial shedding from SMs, reducing SSI risk.

Therefore, in this study, we investigated multiple factors that could influence SM bioburdens, such as SM type, speaking during surgery, and face washing. We aimed to increase our understanding of SSI and provide some additional information regarding the use of SMs during surgeries.

\section{Methods}

\section{Location, personnel, and study design}

The study design was approved by the appropriate ethics review board. The study was performed in the operating room of cleanliness class 100 in a grade IIIA hospital in China. Mask samples were collected from orthopedic surgeons in TJA. The study team consisted of six surgeons, two students, and a microbiologist. The SMs were used in the surgical procedures of TJA. After the surgeries, the SMs were placed into sterile bags and submitted to the researcher. The periphery of the SMs was wiped off, and the surfaces of the SMs were then cut into an average of three parts, and an impression was made on the sterile agar plate (Tryptic Soy Agar Medium) on a clean bench and incubated for 48 hours in a humid aerobic atmosphere at $37^{\circ} \mathrm{C}$. The colony-forming units (CFUs) were then counted. In this experiment, a single blind was used, wherein the student did not know which group the SMs were from.

\section{SM types}

Three different types of masks were used in this study. Mask A is a type of medical mask with one filter screen. Mask B is another type of medical mask with two filter screens. Mask C is a reusable cloth mask, usually washed at intervals of several days. The microstructure of SMs was observed using a scanning electron microscope (SEM). The pore size and porosity were counted using Image J v1.8.0 (National Institutes of Health, Bethesda, MD, United States).

A TSI Automated Filter Tester 8130 (TSI Incorporated) was used to test the filter efficiency and airflow resistance according to PRC National Standard GB/19083-2010 (ICAS Certification \& Testing Group, "Technical requirements for a protective face mask for medical use"). A $0.075 \mu \mathrm{m}$ sodium chloride aerosol generated from salt water solution was used. Filter efficiency and airflow resistance were tested at an $85 \mathrm{~L}$ per minute airflow. The test was performed in two conditions: SMs underwent temperature pretreatment or no pretreatment. The condition of temperature pretreatment was that first SMs were placed in a test chamber at $70 \pm 3{ }^{\circ} \mathrm{C}$ for 24 hours and then at $-30 \pm 3{ }^{\circ} \mathrm{C}$ for 24 hours.

\section{Speaking and no speaking}

We evaluated whether speaking can affect the bioburdens of SMs. Before surgical procedures, the surgeons washed their faces with sterile water, irrigated their nose with a sterile saline solution, and rinsed their mouth with medical mouthwash. Then, in the speaking group, the surgeons were required to recite step-by-step surgical procedures 
during operation. In the non-speaking group, the surgeons were required to speak as few words as possible during the operation. After the surgeries, the SMs were collected, and an impression was made on the sterile agar plate for the following culture and bioburden analysis.

\section{Face-washing or no face-washing}

An additional factor investigated in this study was whether face cleanliness could influence SM bioburden. The surgeons washed their face before the process of surgeries with aseptic water for 10 seconds. The surgeons were required to speak as few words as possible during operation. After the surgeries, the SMs were collected, and an impression was made on the sterile agar plate for the following culture and bioburden analysis.

\section{Statistical analysis}

The results were expressed as the mean \pm standard deviation. Statistical differences were analyzed using one-way analysis of variance. $\mathrm{P}<0.05$ indicates a significant difference.

\section{Results}

\section{Bioburdens of different types of SMs}

Representative SEM images of SMs are depicted in Figure 1. Masks $\mathrm{A}, \mathrm{B}$, and $\mathrm{C}$ had a different pore size and porosity. Mask A possessed the highest pore size and porosity $(\mathrm{P}<0.05)$ (Figure 1B,C). Mask B possessed the lowest porosity $(\mathrm{P}<0.05)$ (Figure 1B). Filter efficiency and airflow resistance are depicted in Figure 2. Mask B possessed the highest filtering efficiency and lowest airflow resistance, which means mask B performed best in blocking airborne particles and provided the best air permeability, enabling the surgeons to breathe freely. On the contrary, mask C possessed the lowest filtering efficiency and highest airflow resistance, meaning it was the worst in blocking airborne particles and in air permeability, causing breathing difficulties in surgeons.

For performing the bioburden assay, bacterial contamination has been used as an adjunct measure of SSI, commonly measured using airborne or settled CFU counts (8). Thus, the number of CFUs from SMs was counted in this present study. Statistically, the number of CFUs from mask B was the lowest with significance, which indicated that mask B was the best in preventing bacterial shedding from personnel. The number of CFUs from mask A was highest, suggesting that mask A provided the poorest protection.

Overall, mask B provided the best protection and enabled surgeons to breathe smoothly; therefore, it should be highly recommended in surgery. Mask A performed worst in blocking bacterial shedding and should not be used in operating rooms. Regarding mask $\mathrm{C}$, it had the highest air flow resistance, which might obstruct comfortable breathing in surgeons. Additionally, mask $\mathrm{C}$ could not block bacterial shedding as effectively as mask B. Notably, mask C was newly laundered, while in most cases, the surgeons did not wash their cloth masks every day.

\section{Speaking and mask bioburdens}

Bacterial shedding that could be restrained by the SM is mainly derived from the face skin and respiratory tract (8). Previous studies have demonstrated that fewer bacteria are expelled from the respiratory tract if the surgeon stays quiet and that avoiding speaking can decrease bacterial dispersion (16-19). However, some researchers hold different views and have shown that wearing masks during quiet breathing could lead to more bacterial shedding (20). Overall, whether speaking can influence bacterial shedding is still controversial. To the best of our knowledge, no previous study has identified whether speaking could influence mask bioburdens.

In this present study, we detected the mask bioburdens in both speaking and no-speaking situations. We demonstrated that CFUs from no-speaking masks were significantly lower than those from speaking masks $(\mathrm{P}<0.05)$ (Figure 3), indicating that unnecessary talking should be restricted in surgery.

\section{Washing face and mask bioburdens}

As the skin's surface is covered with bacteria, patients are encouraged to prophylactically decontaminate their skin before surgery, helping to reduce the risk of SSI. Specifically, washing the face has been recommended in preventing SSI in patients (21).

However, there is no previous study focusing on the relationship between SM bioburdens and face cleanliness. In this study, we examined the mask's bioburdens before and after washing the face. Washing the face resulted in significantly declined CFU numbers on the surgeon's face, but not in the masks (Figure 4).

\section{Discussion}

People often tend to skip steps in daily routines, even in 

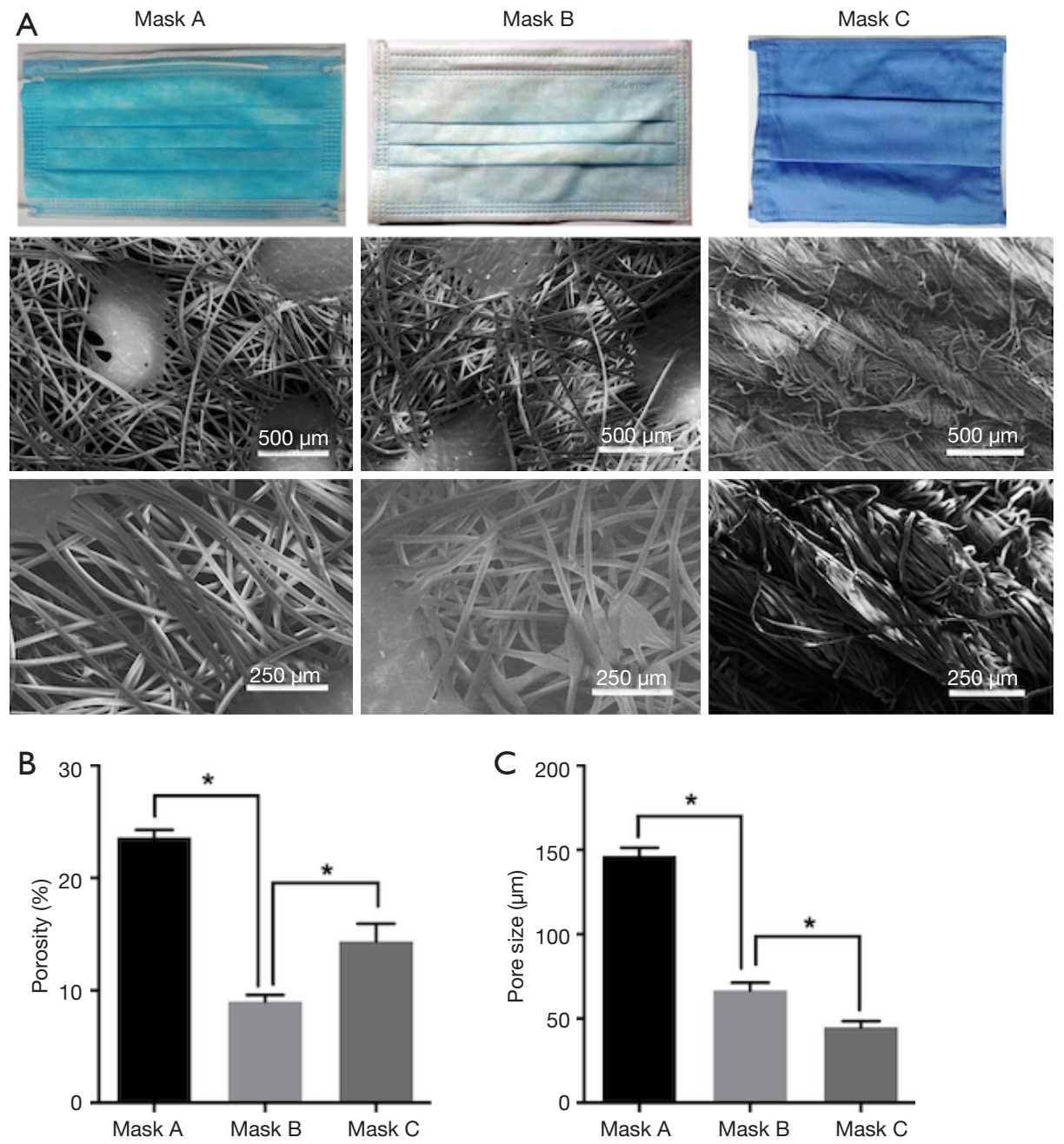

Figure 1 Different mask properties. (A) The scanning electron microscope of masks A, B, and C. (B) Mask porosity. (C) Mask pore size. *, $\mathrm{P}<0.05$.
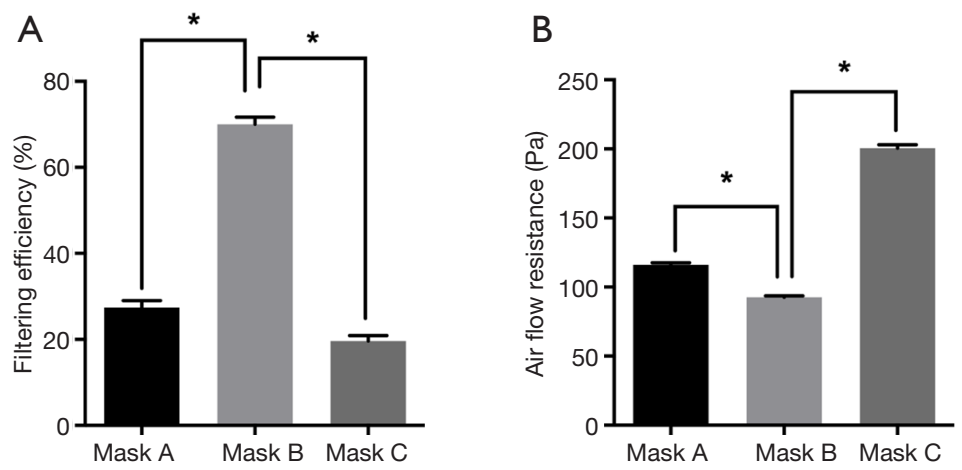

Figure 2 Mask filter efficiency (A) and airflow resistance (B). *, $\mathrm{P}<0.05$. 
A

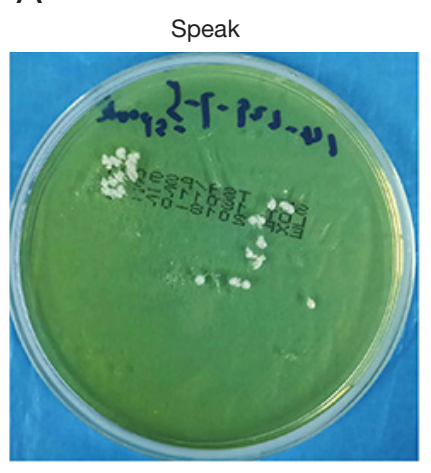

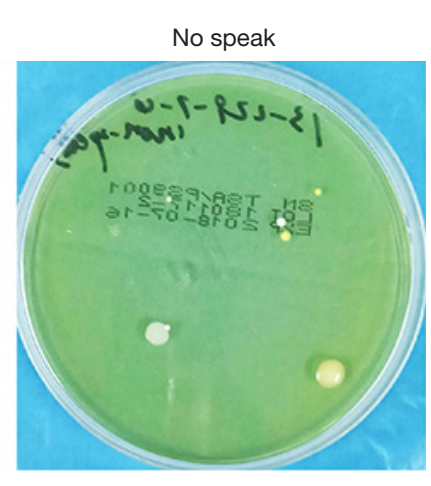

B

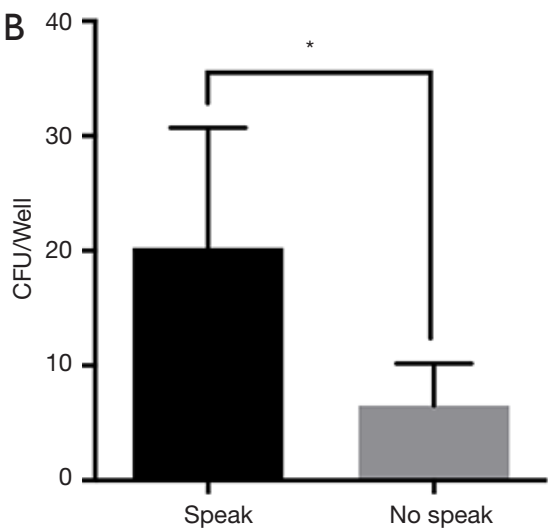

Figure 3 Representative CFUs of masks from speaking and no speaking (A), and statistical results (B). * $\mathrm{P}<0.05$. CFU, colony-forming unit.
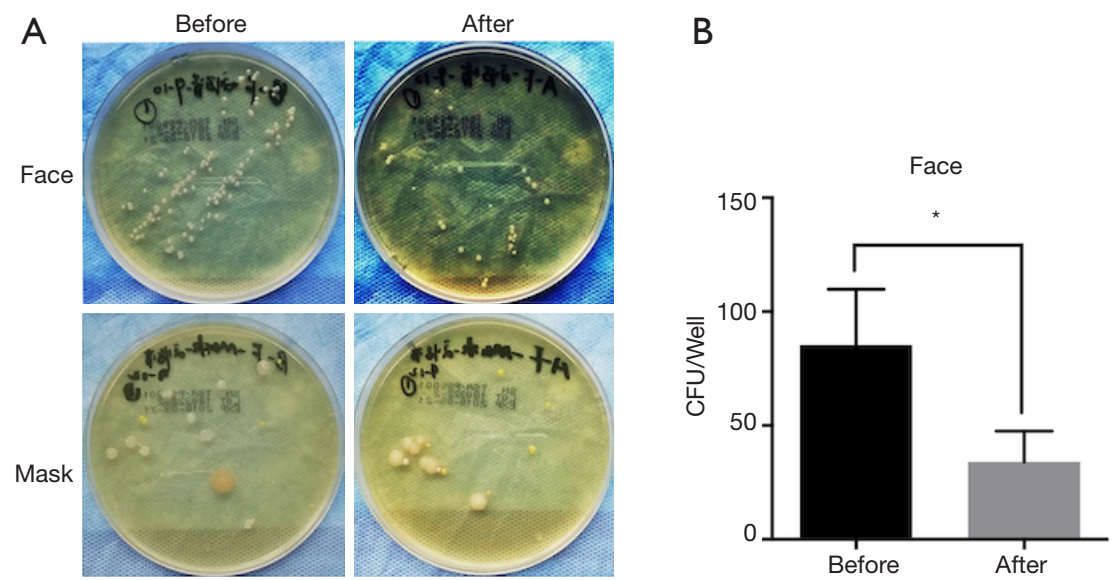

C

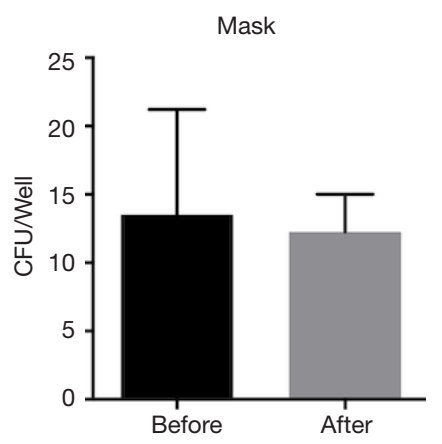

Figure 4 Representative CFUs of faces and masks from face-washing and no face-washing (A), and statistical results (B,C). * $\mathrm{P}<0.05$. CFU, colony-forming unit.

important fields such as surgery. In the operation room, surgical attire items are often neglected and may contribute to SSI occurrence $(22,23)$. SMs have been standard operating apparel since the beginning of the 20th century, although their efficacy has always been controversial (8). There remains a significant lack of organizational consensus, even in today's highly infection-control conscious environment, as to how often the surgeon should change a new mask and which SM to select among the different types. In our previous study, we identified that masks with prolonged wearing time present a potential source of bacterial shedding and transmission of infection in surgery (15). At least in China, in most cases, a surgeon would wear the same SM for the entire day, sometimes wearing the same mask for several surgeries. Since the theory of aseptic technique is founded on the premise that a reduction in bacterial contamination will reduce the prevalence of SSI, identification of key influencing factors that may affect the bioburdens of SMs is of utmost importance and urgency. In this study, we investigated the factors that may influence the bioburdens of SMs and present some recommendations that may help decrease SSI incidence.

First, we evaluated the efficacy of different types of SMs in preventing microbial shedding from personnel. Different masks had diverse pore sizes and porosity (Figure 1). Mask B possessed the lowest porosity and moderate pore size, mask A had the largest porosity and pore size, while mask C had moderate porosity and the smallest pore size. We found no linear relation between porosity and pore size. Regarding filtering efficiency, which is associated with blocking bacterial shedding, mask B performed best in blocking airborne particles (Figure 2). 
A

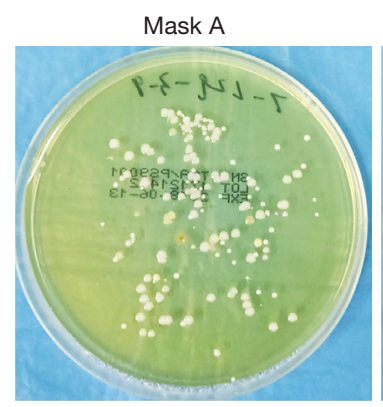

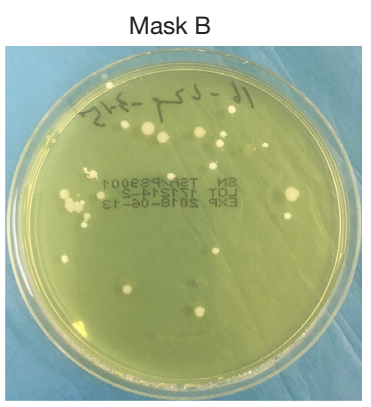
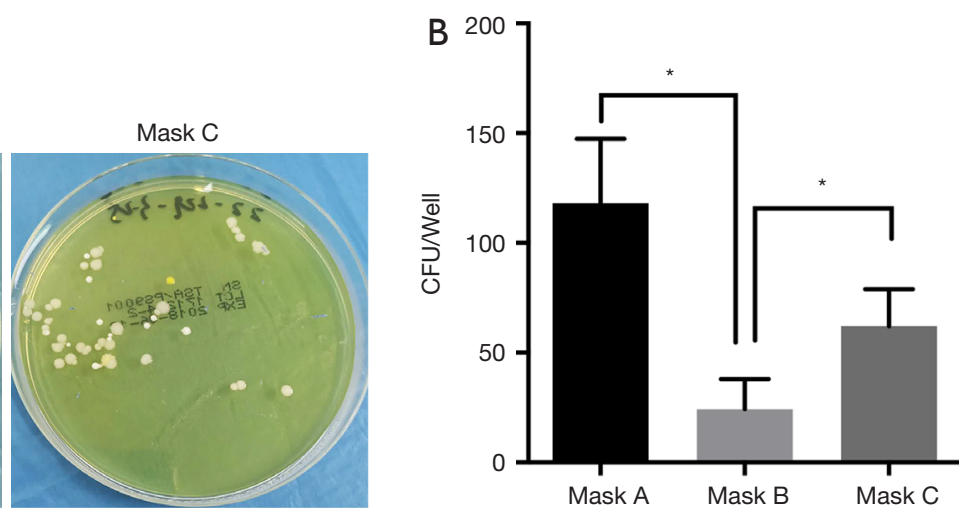

Figure 5 Representative CFUs of different masks (A) and statistical results (B). *, $\mathrm{P}<0.05$. CFU, colony-forming unit.

Furthermore, in the bioburdens assay, mask B had the lowest CFU number, indicating that mask B performed best in restraining bacterial shedding from personnel (Figure 5). Mask A performed worst in blocking bacterial shedding. Concerning airflow resistance, which is associated with surgeons' breathing, mask B showed the best air permeability, allowing surgeons to breathe freely.

On the contrary, mask $\mathrm{C}$ showed the highest airflow resistance and the worst air permeability, which could cause the surgeons to have difficulty in breathing. Overall, mask $\mathrm{B}$ was found to perform best in protection and enabling surgeons to breathe; therefore, it should be recommended for use in surgery. Mask A performed worst in blocking bacterial shedding and should not be used in operating rooms. As mask $\mathrm{C}$ had the highest airflow resistance, the surgeons might not be able to breathe freely when using it. Also, mask C could not block bacterial shedding as effectively as mask B. Notably, mask C used in this study were newly laundered, while in most cases, surgeons do not wash their cloth masks daily.

Furthermore, we investigated the effect of speaking on the bioburdens of SMs. Previous studies have demonstrated that the respiratory tract is a source of bacterial shedding in the operation room (8), while fewer bacteria would be expelled from the respiratory tract if the surgeon remains quiet (16-19). However, whether speaking can influence mask bioburdens is not clear. In this present study, we demonstrated that the bioburden from the no-speaking masks was significantly lower than that from speaking masks (Figure 3), indicating that masks were beneficial in reducing bacterial counts during talking, which was in line with the results of the previous study (24). Therefore, unnecessary talking by surgeons should be restricted in surgery.
Another source of bacterial shedding in the operation room is the skin surface, and washing the face has been recommended to prevent SSI in patients (21). However, to the best of our knowledge, no previous study has identified the relationship between SM bioburden and the wearers' facial cleanliness. In this study, we showed that washing the face led to a significantly reduced CFU number on the surgeon's face, but the same was not true for the SMs (Figure 4).

There were certain limitations to this study. We have not investigated the relationship between the bioburdens of SMs and the incidence of SSIs. However, similar to the statement in our previous study, we performed this study based on the understanding that the theory of aseptic technique is founded on the premise that a reduction in bacterial contamination will reduce the prevalence of SSI. Moreover, as the saying goes: "Do not think any virtue trivial, and so neglect it; do not think any vice trivial, and so practice it" (15). Measures to control SSIs are of utmost importance and should be valued. We hope this present study can increase the knowledge about SSIs and focus more attention on their related risk factors. Future studies are warranted to identify the bacterial organisms that constitute the bioburden and potential clinical impact, if any, on the development of SSIs.

\section{Conclusions}

We determined that multiple factors are involved in the bioburdens of SMs, such as SMs types, speaking, and face cleanness. Based on the results of the present study, several conclusions can be drawn here: (I) mask type is a crucial factor that has a direct relationship with mask bioburdens; therefore, surgeons should be more prudent in selecting SMs. Masks with high filtering efficiency and low airflow resistance 
should be recommended. (II) Speaking can increase the mask bioburdens; therefore, unnecessary speaking should be avoided in surgery. (III) Washing the face can reduce the bioburdens on the face, but not the mask.

\section{Acknowledgments}

Funding: This work was supported by the National Natural Science Foundation for Youths (Grant No. 81601958), Shanghai Sailing Program (Grant No. 16YF1407500), Shanghai Jiao Tong University Medical and Engineering Cross Fund (Grant No. YG2015QN41), Shanghai Clinical Medical Center (Grant Number 2017ZZ01023), and Shanghai Municipal Key Clinical Specialty.

\section{Footnote}

Conflicts of Interest: The authors have no conflicts of interest to declare.

Ethical Statement: The authors are accountable for all aspects of the work in ensuring that questions related to the accuracy or integrity of any part of the work are appropriately investigated and resolved. The study design was approved by the appropriate ethics review board.

\section{References}

1. Tanner J, Dumville JC, Norman G, et al. Surgical hand antisepsis to reduce surgical site infection. Cochrane Database Syst Rev 2016;(1):CD004288.

2. Allegranzi B, Bischoff P, de Jonge S, et al. New WHO recommendations on preoperative measures for surgical site infection prevention: an evidence-based global perspective. Lancet Infect Dis 2016;16:e276-87.

3. Scott RD. The DirecT MeDical cosTs of HealthcareAssociated Infections in U.S. Hospitals and the Benefits of Prevention. 2009.

4. Markel TA, Gormley T, Greeley D, et al. Hats Off: A Study of Different Operating Room Headgear Assessed by Environmental Quality Indicators. J Am Coll Surg 2017;225:573-81.

5. Tammelin A, Ljungqvist B, Reinmuller B. Single-use surgical clothing system for reduction of airborne bacteria in the operating room. J Hosp Infect 2013;84:245-7.

6. Chauveaux D. Preventing surgical-site infections: measures other than antibiotics. Orthop Traumatol Surg Res 2015;101:S77-83.

7. McHugh SM, Corrigan MA, Hill AD, et al. Surgical attire, practices and their perception in the prevention of surgical site infection. Surgeon 2014;12:47-52.

8. Salassa TE, Swiontkowski MF. Surgical attire and the operating room: role in infection prevention. J Bone Joint Surg Am 2014;96:1485-92.

9. Weaver GH. Droplet Infection and Its Prevention by the Face Mask. J Infect Dis 1919;24:218-30.

10. McLure HA, Talboys CA, Yentis SM, et al. Surgical face masks and downward dispersal of bacteria. Anaesthesia 1998;53:624-6.

11. Tunevall TG. Postoperative wound infections and surgical face masks: A controlled study. World J Surg 1991;15:383-7.

12. Eisen DB. Surgeon's garb and infection control: what's the evidence? J Am Acad Dermatol 2011;64:960.e1-20.

13. Sellden E. Is routine use of a face mask necessary in the operating room? Anesthesiology 2010;113:1447.

14. Datta R. Use of surgical facemasks in the operation theatre: Effective or habit? Med J Armed Forces India 2010;66:163-5.

15. Zhiqing L, Yongyun C, Wenxiang C, et al. Surgical masks as source of bacterial contamination during operative procedures. J Orthop Translat 2018;14:57-62.

16. Shooter RA, Smith MA, Hunter CJ. A study of surgical masks. Br J Surg 1959;47:246-9.

17. Hare R, Thomas C. The transmission of Staphylococcus aureus. Br Med J 1956;2:840.

18. Mitchell NJ, Hunt S. Surgical face masks in modern operating rooms-a costly and unnecessary ritual? J Hosp Infect 1991;18:239-42.

19. Ritter MA, Eitzen H, French M, et al. The operating room environment as affected by people and the surgical face mask. Clin Orthop Relat Res 1975;(111):147-50.

20. Hirshfeld JW, Laube PJ. Surgical masks: an experimental study. Surgery 1941;9:720-30.

21. Stephen-Haynes J. Infection prevention and control in a community setting. Wound Care in the Community 2014:23.

22. $\mathrm{LiX}, \mathrm{Li} \mathrm{M}, \mathrm{LiJ}$, et al. Glove perforation and contamination in fracture fixation surgeries. Am J Infect Control 2017;45:458-60.

23. Twomey CL, Beitz H, Johnson HB. Bacterial contamination of surgical scrubs and laundering mechanisms: infection control implications. Studies 2010;6:16-21.

24. Doshi RR, Leng T, Fung AE. Reducing oral flora contamination of intravitreal injections with face mask or silence. Retina 2012;32:473-6.

Cite this article as: Liu Z, Yu D, Ge Y, Wang L, Zhang J, Li H, Liu F, Zhai Z. Understanding the factors involved in determining the bioburdens of surgical masks. Ann Transl Med 2019;7(23):754. doi: 10.21037/atm.2019.11.91 